\title{
CX43 Expression in Colonic Adenomas and Surrounding Mucosa Is a Marker of Malignant Potential
}

\author{
ALEN BIŠĆANIN ${ }^{1}$, NEVEN LJUBIČIĆ ${ }^{1}$, MARKO BOBAN $^{2,3}$, DRINKO BALIČEVIĆ ${ }^{4}$, IVANA PAVIĆ ${ }^{4}$, \\ MIRELA MAVER BIŠĆANIN ${ }^{5}$, IVAN BUDIMIR ${ }^{1}$, ZDRAVKO DOROSULIC ${ }^{1}$ and MARKO DUVNJAK ${ }^{1}$ \\ ${ }^{1}$ Division of Gastroenterology, Department of Internal Medicine, University Hospital Centre Sestre Milosrdnice, \\ University of Zagreb School of Medicine and University of Zagreb School of Dental Medicine, Zagreb, Croatia; \\ ${ }^{2}$ Department of Cardiology, University Hospital Thalassotherapia Opatija, \\ Medical Faculty University of Rijeka, Opatija, Croatia; \\ ${ }^{3}$ Department of Internal Medicine, University Hospital Osijek, \\ Medical Faculty JJ Strossmayer University of Osijek, Osijek, Croatia; \\ ${ }^{4}$ Ljudevit Jurak University Department of Pathology, University Hospital Centre Sestre Milosrdnice, \\ University of Zagreb School of Medicine and University of Zagreb School of Dental Medicine, Zagreb, Croatia; \\ ${ }^{5}$ Department of Prosthodontics, Zagreb Dental Clinic, Zagreb, Croatia
}

\begin{abstract}
Background/Aim: Colorectal cancer is a major public health problem. The adenoma-carcinoma sequence offers potential for screening and surveillance. We tested the clinical behavior and diagnostic utility of connexin 43 (CX43) in connection with pathohistological risk. Patients and Methods: Immunohistochemical expression of CX43 in colonic adenomas and surrounding mucosa from 87 patients was determined. Results: CX43 expression was higher in mucosa surrounding adenomas with high-grade dysplasia $(p=0.047)$, larger adenomas $(p=0.015)$ and villous adenomas ( $p=0.02)$. No difference of CX43 expression in adenomas according to grade of dysplasia was found $(p=0.87) . C X 43$ expression in adenomas was dependent on the patient's hemoglobin level ( $p=0.002)$, family history of colorectal cancer $(p=0.009)$ and statin therapy $(p=0.049)$. Conclusion: CX43 expression in mucosa surrounding adenoma could be an additional factor indicative of malignant potential. CX43 expression in colonic adenoma seems to be closely related to family history of colorectal cancer, statin therapy and hemoglobin level.
\end{abstract}

Correspondence to: Alen Bišćanin, MD, Ph.D., Department of Internal Medicine, University Hospital Centre Sestre Milosrdnice, University of Zagreb School of Medicine and University of Zagreb School of Dental Medicine, Vinogradska cesta29, Zagreb10000, Croatia. Tel: +385 13787455, Fax: +385 1375625, e-mail: alen.biscanin@gmail.com

Key Words: CX43, malignant potential of adenoma, colorectal cancer.
The process of colorectal carcinogenesis is the ideal model for studying the course of malignant transformation, considering well-defined steps in cancer development. It is widely accepted that the majority of colorectal carcinomas (CRC) arise from adenomas (1). Genetics, external factors and their combinations are crucial for the growth of colonic polyps (2).

The adenoma-carcinoma sequence in CRC has been repeatedly and unequivocally confirmed (3-5). Much effort has been put into prevention of CRC using fecal occult blood test and colonoscopy with polypectomy (6). CRC risk estimation encompasses endoscopic examination based on size, number and pathohistological characteristics of polyps, patient age, inflammatory bowel disease, family history of CRC and genetic predisposition. Postpolypectomy surveillance colonoscopies account for $20 \%$ of all colonoscopic procedures and are becoming a burden in CRC prevention, especially considering that only a small percentage of adenomas will actually become malignant (1).

Connexins are a family of transmembrane proteins that assemble to form vertebrate gap junctions (GJs). Their key role is in regulating cell growth and apoptosis (7-9), providing intercellular communication through and independently of GJs (8-10). GJs are molecular complexes consisting of hundreds of channels on the membranes of two neighboring cells. Each single channel is composed of two subunits on the neighboring cells, called connexons. Connexons are composed of six connexins (7). Connexin 43 (CX43) is the most common member of the connexin family. In the gastrointestinal tract, CX43 is present in all mucosal layers except the longitudinal muscle layer, forming connections among the various cell types $(11,12)$. In the 
Table I. Clinicopathological characteristics of patients and adenomas included in study.

\begin{tabular}{|c|c|c|}
\hline & \multicolumn{2}{|c|}{ Dysplasia } \\
\hline & Low-grade, n (\%) & High-grade, $\mathrm{n}(\%)$ \\
\hline \multicolumn{3}{|l|}{ Gender } \\
\hline Female & $9(20.9 \%)$ & $12(27.3 \%)$ \\
\hline Male & $34(79.1 \%)$ & $32(72.7 \%)$ \\
\hline \multicolumn{3}{|l|}{ Adenoma, histological type* } \\
\hline Tubular & $30(69.8 \%)$ & $15(34.1 \%)$ \\
\hline Villous \& tubulovillous & $13(30.2 \%)$ & $29(65.9 \%)$ \\
\hline \multicolumn{3}{|l|}{ Adenoma location } \\
\hline Right colon & $13(30.2 \%)$ & $12(27.3 \%)$ \\
\hline Left colon & $30(69.8 \%)$ & $32(72.7 \%)$ \\
\hline Positive fecal occult blood test & $19(54.3 \%)$ & $24(75.0 \%)$ \\
\hline Constipation & $11(25.6 \%)$ & $14(31.8 \%)$ \\
\hline Use of laxatives & $10(23.3 \%)$ & $14(31.8 \%)$ \\
\hline \multicolumn{3}{|l|}{ Nutrition type } \\
\hline Mediterranean & $3(7.0 \%)$ & $3(6.8 \%)$ \\
\hline Continental & $35(81.4 \%)$ & $31(70.5 \%)$ \\
\hline Cigarette smoking & $14(32.6 \%)$ & $13(29.5 \%)$ \\
\hline Alcohol consumption & $17(39.5 \%)$ & $12(27.3 \%)$ \\
\hline Aspirin therapy & $10(23.3 \%)$ & $9(20.5 \%)$ \\
\hline Statin therapy & $14(32,6 \%)$ & $11(25,0 \%)$ \\
\hline Positive family history of colon cancer & $7(16.3 \%)$ & $8(18.2 \%)$ \\
\hline Adenoma size estimated on endoscopy $(\operatorname{mean} \pm \mathrm{SD}, \mathrm{mm})$ & $10.77 \pm 3.18$ & $15.91 \pm 8.7^{*}$ \\
\hline Hemoglobin level $(\mathrm{mg} / \mathrm{dl})$ & 14.7 & $13.8^{*}$ \\
\hline
\end{tabular}

*Significantly different at $p<0.05$.

process of colorectal carcinogenesis, CX43 is a player in the wingless/integrated (WNT) signaling pathway, and betacatenin enhances CX43 transcription. Regulation of betacatenin feedback by CX43 is possible and CX43 negatively regulates WNT signaling pathway by this mechanism (1315). Connexins in carcinogenesis may be classified as conditional tumor suppressors, depending on the connexin isoform, cancer type and stage (3).

To the best of our knowledge, the diagnostic value of CX43 in colonic adenomas and the surrounding mucosa has not been well defined. The aim of our study was to determine the CX43 expression pattern in colonic adenomas and surrounding colonic mucosa and its potential significance for the malignant potential of adenoma.

\section{Patients and Methods}

This was a prospective non-randomised study performed in a tertiary medical center intended to serve a catchment population of 300,000 people. Consecutive patients scheduled for CRC prevention colonoscopy, with polypectomy, in the period between April 2012 until September 2013 were included in the study after giving their written informed consent. The study was approved by University Hospital Center Sestre Milosrdnice Ethical Committee, approval number EP-5816/11-2, in line with good clinical practice. Endoscopic procedures were performed with standard equipment (video colonoscope CF-Q165I; Olympus, Tokyo, Japan) by well-trained endoscopists with at least 5 years' experience. Polyp dimension was determined by comparing with open biopsy forceps or a marked guide wire and by pathologists' measurements (16). Patients with inflammatory bowel disease, previously diagnosed malignant disease, malignancy detected during endoscopy or those not willing to provide the informed consent were excluded from the study. The identity of all included patients was confidential.

For each patient, samples of one adenoma and two biopsies from the surrounding colonic mucosa (approximately $1 \mathrm{~cm}$ from the adenoma cut edge) following polypectomy were taken. Surrounding colonic mucosa selected for immunohistochemical studies did not reveal significant pathohistological changes under light microscopy examination. All patients included in the study filled out a detailed questionnaire about their dietary habits, bodily functions, medications and complete medical history.

Specimens of adenomas and additional fragments of surrounding colonic mucosa (not adjacent to adenoma) were fixed in $10 \%$ buffered formalin, paraffin embedded, cut at 5- $\mu \mathrm{m}$ thickness and routinely stained with hematoxylin and eosin. Adenoma type and dysplasia grade for all adenoma specimens were histologically determined according to the World Health Organization 2010 classification (17).

Deparaffinization and immunohistochemical staining was performed according to the Microwave Streptavidin ImmunoPeroxidase (MSIP) protocol on a DAKO Tech-Mate TM Horison automated immunostainer (DAKO, Copenhagen, 


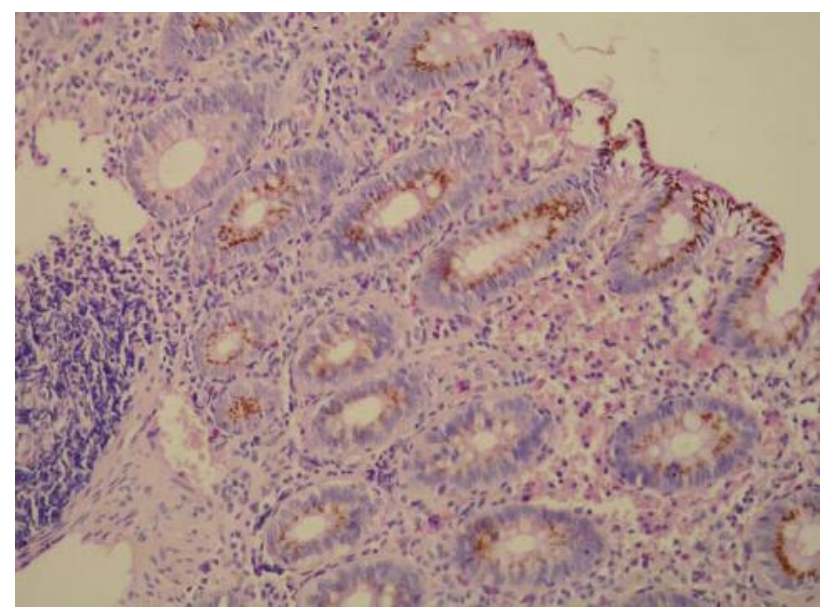

Figure 1. Colonic mucosa surrounding adenomas was found to exhibit finely granular membranous expression of connexin 43 (magnification $\times 200$ ).

Denmark). We used polyclonal goat IgG antibody to connexin 43 (SC6560, dilution 1:300; Santa Cruz Biotechnology, Inc., Dallas, TX, USA). Immunohistochemical expression of CX43 was evaluated in the epithelium in all adenomas and in the epithelium in additional colonic mucosa fragments, negative controls were included with omission of the primary antibodies. Two experienced pathologists analyzed CX43 expression on the whole cut surface on slides using a light microscope (CX22LED; Olympus Europa GMBH, Hamburg, Germany) under low ( $\times 40)$ and medium $(\times 200)$ magnification. The final CX43 expression score was determined as average count of cells with positive membranous staining by two pathologists on a 2-point scale: negative, $<10 \% \mathrm{CX} 43$-positive cells, and positive, $\geq 10 \% \mathrm{CX} 43$ positive cells. CX43 expression was correlated with adenoma pathohistological characteristics as well as patient characteristics.

The analyzed samples were allocated on the basis of pathohistological grading of epithelial dysplasia into groups with high-grade and low-grade dysplasia. A comparison of clinical, pathohistological, endoscopical and laboratory characteristics between these two groups of patients was made.

Statistical analysis. The whole patient population and studied groups were analyzed with descriptive statistics and results are presented as means combined with standard deviations. Categorical variables are presented as counts (percentages) and evaluated using Chi-square tests. Laboratory, endoscopic, pathological and remaining numeric data were tested for differences by MannWhitney $U$-test. Associations of CX43 with studied clinical parameters were evaluated by Spearman's rho. The diagnostic value of CX43 for the malignant potential of adenoma was tested with receiver operating curve analyses (ROC), using the criterion corresponding with highest Youden index. $p$-Values less than 0.05 were considered significant. Statistical analyses were carried out by an experienced statistician using Statistica v.10 for Windows (StatSoft inc., Tulsa, OK, USA), IBM-SPSS 12 v. 20 (IBM corp., Chicago, IL, USA) and MedCalc v. 12.2 for Windows (MedCalc Software corp., Ostend, Belgium).

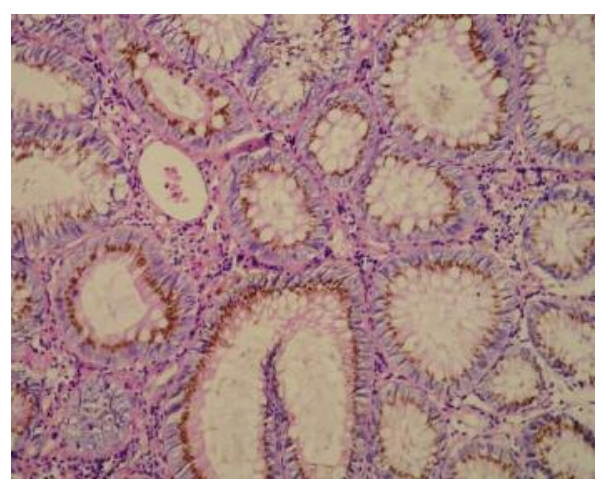

Figure 2. Adenoma with low-grade dysplasia. Colonic adenomas were found to exhibit finely granular membranous expression of connexin 43 (magnification $\times 200$ ).

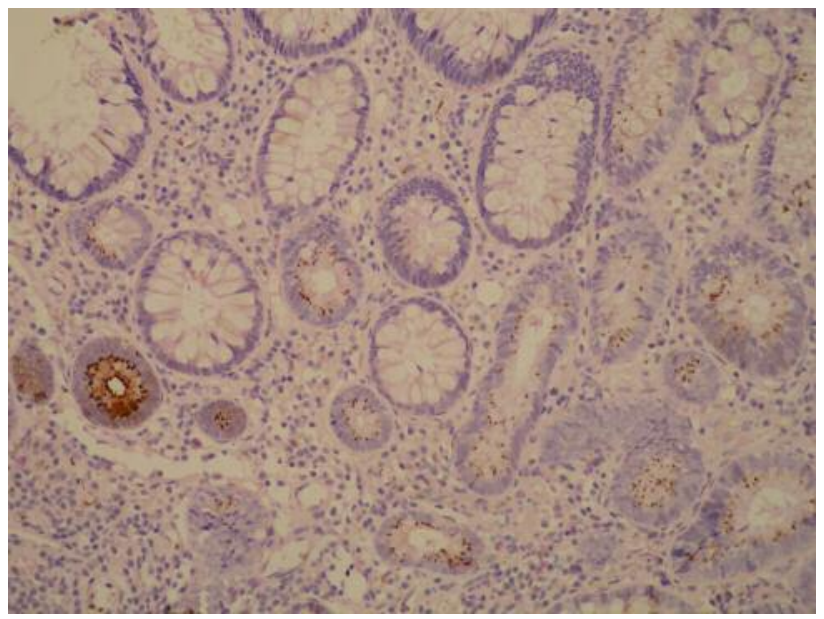

Figure 3. Adenoma with low-grade dysplasia. Adenomas were usually found to exhibit simultaneous fine granular membranous and coarsely granular cytoplasmic expression of connexin 43 (magnification $\times 200$ ).

\section{Results}

A total of 87 patients, median age 66 (range=24-82) years, scheduled for CRC prevention colonoscopy and polypectomy of colonic adenomas were included in the study: 43 patients had low-grade adenoma/dysplasia and 44 patients high-grade adenoma/dysplasia. Two biopsies of mucosa-surrounding adenomas were taken from all patients. The main clinicopathological characteristics of patients and adenomas included in this study are shown in Table I.

Adenomas with low-grade dysplasia were smaller (10.8 mm vs. $15.9 \mathrm{~mm} ; p<0.01)$, more often having a tubular-type histology $(69.8 \%$ vs. $34.1 \%, p<0.05)$. No difference between 
the groups of patients or adenomas with low- or high-grade dysplasia was found regarding sex, age, polyp location, positivity for fecal occult blood test, presence of constipation or laxative intake, cuisine type, cigarette smoking, alcohol consumption, aspirin or statin therapy, or positive family history of CRC. The hemoglobin level was significantly higher in patients with adenoma with low-grade dysplasia $(14.7 \mathrm{~g} / \mathrm{dl} v s .13 .8 \mathrm{~g} / \mathrm{dl}, p=0.014)$.

CX43 expression in colonic adenomas and surrounding colonic mucosa was found in epithelial cells and the epithelium of the crypts. Immunohistochemical reaction to CX43 in colonic mucosa was located exclusively in the membrane of epithelial cells, while in adenomas it was also found in the cytoplasm (Figures 1-3). Mucosal and adenoma membranous reaction was finely granular, located most often between apical parts of the cells, less in the middle of the cell, and was rarely found on the luminal side of the membrane of cells (Figures 1-2). Cytoplasmic reaction in adenomas was coarsely granular (Figure 3 ).

There was no significant difference in CX43 expression in adenomas regarding the grade of dysplasia $(p=0.87)$.

Adenomas of the 15 patients with a positive family history of CRC among first-degree relatives were all CX43-positive, in contrast to patients with a negative family history of CRC (65.3\% CX43-positive), regardless of endoscopic and histopathological features of adenomas $(p=0.009)$.

ROC analyses for CX43 expression in colonic adenomas and patient hemoglobin were significant: area under the curve $(\mathrm{AUC})=0.729[95 \%$ confidence interval $(\mathrm{CI})=0.623-0.819], p=0.0002$. The cut-off for positive expression of CX43 was found with hemoglobin $>14 \mathrm{~g} / \mathrm{dl}$, the ROC curve having a sensitivity of $75.8 \%(95 \%$ $\mathrm{CI}=63.3-85.8 \%)$, specificity of $72 \% \quad(95 \% \quad \mathrm{CI}=50.6-$ $87.9 \%)$, with a positive likelihood ratio (LR) of $2.71(95 \%$ $\mathrm{CI}=2.0-3.6)$ and a negative $\mathrm{LR}$ of 0.34 (95\% $\mathrm{CI}=0.2-0.7)$. CX43 expression was lower in adenomas from patients under statin therapy [odds ratio $(\mathrm{OR})=2.694,95 \%$ $\mathrm{CI}=1.002-7.242, p=0.049]$.

Furthermore, CX43 expression in colonic adenomas was not dependent on patient's sex, history of alcohol consumption, cigarette smoking, aspirin or laxative therapy, polyp location, type of histology or type of nutrition. There was no correlation between CX43 expression and adenoma size measured endoscopically ( $p=0.493)$, size of histological specimen $(p=0.496)$ or patient age $(p=0.102)$.

Significantly higher CX43 expression was found in colonic adenomas when compared to the surrounding colonic mucosa $(71.3 \%$ vs. $51.7 \%, p=0.002)$.

Mucosa surrounding adenomas with high-grade dysplasia more frequently had a positive CX43 reaction when compared to those with low-grade dysplasia $(65.1 \% \mathrm{vs}$. $37.2 \%$; $\mathrm{OR}=2.574,95 \% \mathrm{CI}=1.013-6.540, p=0.047)$. Mucosa surrounding adenomas with villous histology were also significantly more often CX43-positive (65.8\% vs. $40.8 \%$, $p=0.02)$. Mucosa surrounding endoscopically estimated larger adenomas was more often CX43-positive $(p=0.015)$; this was reflected by a similar trend for larger adenomas measured from pathological specimens $(p=0.072)$. ROC analyses for CX43 expression in colonic mucosa and endoscopically estimated adenoma size were significant: AUC $=0.652(95 \% \mathrm{CI}=0.542-0.751, p=0.0089)$. A cut-off for positive expression of CX43 was found for adenoma $>10 \mathrm{~mm}$, with the ROC curve having a sensitivity of $60 \%$ (95\% CI=44.3-74.3\%), specificity of $66.7 \%(95 \% \mathrm{CI}=50.5-$ $80.4 \%)$, a positive LR of $1.8(95 \% \mathrm{CI}=1.3-2.5)$ and a negative LR of 0.6 (95\% CI=0.3-1.00). ROC analyses for CX43 expression in colonic mucosa and size of adenoma measured on pathological specimen were not significant, although they indicated a statistical trend (AUC $=0.612,95 \%$ $\mathrm{CI}=0.502-0.715 ; p=0.0614)$.

CX43 expression in colonic mucosa was also not dependent on patient sex, family history of CRC, alcohol consumption, laxative, aspirin or statin therapy, cigarette smoking, nutrition type, polyp location or hemoglobin level. There was no correlation between mucosal CX43 expression and patient age $(p=0.78)$.

Complications of endoscopic resection of adenomas occurred in two patients $(2.2 \%)$. In one patient, bleeding was noticed on the fifth postoperative day, and it was solved by clipping hemostasis on repeated colonoscopy. The other patient developed post-polypectomy electrocoagulation syndrome and broad-spectrum antibiotics (ciprofloxacin plus metronidazole) were administered. Complications as a result of additional mucosal biopsy were not seen.

\section{Discussion}

In this study we investigated the diagnostic value of CX43 in colonic adenomas because the importance of CX43 in early stages of colorectal carcinogenesis is not well defined.

Kanczuga-Koda et al. described CX43 expression in colonic adenomas with tubular-type growth and low-grade dysplasia (18). Han et al. later showed a similar pattern of CX43 staining in specimens of high-grade dysplasia at the margin of CRC (13). Relocation of CX43 reflects the beginning of the loss of functional GJs, which starts in cells with the appearance of dysplasia, and continues with the progression of dysplasia to adenocarcinoma (18-20). The cytoplasmic location of connexins enables interaction with other cellular proteins, influencing cellular messenger pathways $(9,10)$. Comparing CX43 expression in adenomas with low-grade dysplasia and high-grade dysplasia, unexpectedly we found no differences. Interestingly, CX43 expression in colonic adenomas was dependent on patient's hemoglobin level, family history of CRC among first-degree relatives and statin therapy. 
It is known that the $C X 43$ gene may be a target for mutated adenomatous polyposis coli (APC) gene/protein, and that the WNT signaling pathway and beta-catenin transcription enhances CX43 transcription, which again has the possibility of beta-catenin feedback regulation $(12,13)$. It is assumed that connexins enter the nucleus and there are hypotheses that connexins act directly on the transcription of genes involved in the regulation of cell growth (20). CX43, thereby, negatively regulates WNT signaling pathway (3).

In our study, CX43 expression was detected in adenomas of all patients with a positive family history of CRC among first-degree relatives, regardless of endoscopic and histopathological features of their adenomas. These data suggest the influence of heritage on CX43 expression, but given the relatively small number of patients, and the fact that APC protein was not investigated in our study, this finding would certainly require further research.

CX43 expression was higher in colonic adenoma of patients with a higher hemoglobin level, and the cut-off for positive expression of CX43 was found with a hemoglobin level $>14 \mathrm{~g} / \mathrm{dl}$. This finding was expected, knowing that tissue oxidation protects CX43 from degradation (21).

CX43 expression was lower in adenoma (not in mucosa) of patients with statin therapy, which could be one possible mechanism by which statins reduce the risk of CRC (22). We found no differences of CX43 expression in colonic adenoma (and mucosa) related to aspirin therapy.

CX43 expression in mucosa surrounding colonic adenoma has not been studied before. Dubina et al. were the first to describe CX43 expression in colonic mucosa using immunohistochemistry (12). We found that the pattern of membranous reactions in colonic mucosa was the same as in adenoma. In contrast to earlier research (11), cytoplasmic location of CX43 in mucosal epithelial cells was not evident. This may be explained by the short half-life of CX43 and the fact that its synthesis and changes in the cell membranes are very intense processes (7).

As in the study of Han et al. (13), mucosal CX43 expression was lower than that in the study of Kanzuga-Koda et al. (11), and later Sirnes et al. (8). This difference can be partially explained by the staining methods being similar but not the same, and the use of different antibodies. However, in our study the number of CX43-positive colonic mucosa epithelial cells dependent on the location where mucosa was sampled. Surprisingly, epithelial cells surrounding adenomas with highgrade dysplasia showed higher CX43 expression. In addition, CX43 expression was higher in the cells surrounding villous and tubulovillous adenomas, and in the mucosa surrounding larger adenomas (cut-off for positive expression of CX43 was size of adenoma $>10 \mathrm{~mm}$ ). Correlation of CX43 expression in cells and tissues with the presence of neighboring neoplastic changes is not new $(14,15)$. Intensification of communication between neoplastic changes and stroma may help metastasis or create preconditions for functional disorders. This phenomenon could also explain the diversity of CX43 expression in epithelial cells of colonic mucosa found in earlier studies, since previously staining was made on 'healthy' mucosa from the margin of resected CRC. A possible bias of our study is the relatively small preparation of mucosa biopsies sampled with biopsy forceps, and the relatively small number of patients, which could affect our final results.

We did not find any correlation of CX43 expression in adenomas and colonic mucosa with history of smoking as an earlier study suggested (23).

Higher CX43 expression in adenomas compared to healthy colonic mucosa clearly suggests an active role of CX43 in the process of carcinogenesis. A shift in CX43 expression from normal to hyperplastic/dysplastic tissue was shown earlier $(24,25)$. Additionally, the finding of higher CX43 expression in the colonic mucosa surrounding advanced colorectal adenomas is very intriguing. Based on our results, we can assume that CX43 acts in the regulation of cell growth and tissue development by exchange of protective ('good Samaritan' effect, 'kiss of life') or harmful ('kiss of death') signals $(26,27)$.

To the best of our knowledge, we are the first to describe CX43 expression in colonic adenomas with high-grade dysplasia and colonic mucosa surrounding adenomas using immunohistochemistry. According to our results, CX43 expression is higher in mucosa surrounding advanced colorectal adenomas. Given the burden of CRC and that a small number of adenomas transform into cancer during a human life cycle, it is crucial to identify patients with a high long-term risk and need surveillance. Based on our results, we conclude that the CX43 expression in colonic mucosa surrounding adenoma could be an additional diagnostic marker for the prediction of the malignant potential of adenoma. CX43 expression was lower in adenoma of patients with statin therapy, which could be a possible mechanism by which statins might reduce the risk of $\mathrm{CRC}$. The association of CX43 expression in adenomas with a positive family history of CRC among first-degree relatives requires further research.

\section{Conflicts of Interest}

Drs. Alen Bišćanin, Neven Ljubičić, Marko Boban, Drinko Baličević, Ivana Pavić, Mirela Maver Bišćanin, Ivan Budimir, Zdravko Dorosulic and Marko Duvnjak have no conflicts of interest or financial ties to disclose.

\section{References}

1 Leslie A, Carey FA, Pratt NR and Steele RJ: The colorectal adenoma-carcinoma sequence. Br J Surg 89: 845-860, 2009.

2 Correa P: Epidemiology of polyps and cancer. In: The Pathogenesis of Colorectal cancer. Morson BC (ed.). WB Saunders, Philadelphia, p. 126, 1978. 
3 Sirnes S, Lind GE, Bruun J, Fykerud TA, Mesnil M, Lothe RA, Rivedal E, Kolberg M and Leithe E: Connexins in colorectal cancer pathogenesis. Int J Cancer 137: 1-11, 2014.

4 Fearon ER and Vogelstein B: A genetic model for colorectal tumorigenesis. Cell 61: 759-767, 1990.

5 Muto T, Bussey HJR and Morson BC: The evolution of cancer of the colon and rectum. Cancer 36: 2251-2270, 1975.

6 Lieberman DA, Holub J, Eisen G, Kraemer D and Morris CD: Utilization of colonoscopy in the United States: results from a national consortium. Gastrointest Endosc 62: 875-883, 2005.

7 Laird DW: The life cycle of a connexin: gap junction formation, removal, and degradation. J Bioenerg Biomembr 4: 311-318, 1996.

8 Sirnes S, Bruun J, Kolberg M, Kjenseth A, Lind GE, Svindland A, Brech A, Nesbakken A, Lothe RA, Leithe E and Rivedal E: Connexin 43 acts as a colorectal cancer tumor suppressor and predicts disease outcome. Int J Cancer 131: 570-581, 2012.

9 Sun Y, Zhao X, Yao Y, Qi X, Yuan Y and Hu Y: Connexin 43 interacts with Bax to regulate apoptosis of pancreatic cancer through a gap junction-independent pathway. Int J Oncol 41: 941-948, 2012.

10 Zhang YW, Kaneda $M$ and Morita I: The gap junctionindependent tumor-suppressing effect of connexin 43. J BiolChem 278: 44852-44856, 2003.

11 Kanczuga-Koda L, Sulkowski S, Koda M, Sobaniec-Lotowska $M$ and Sulkowska M: Expression of connexins 26, 32 and 43 in the human colon - an immunohistochemical study. Folia Histocem Cytobiol 42: 203-207, 2004.

12 Dubina MV, Iatckii NA, Popov DE, Vasil'ev SV and Krutovskikh VA: Connexin 43, but not connexin 32, is mutated at advanced stages of human sporadic colon cancer. Oncogene 21: 4992-4996, 2002.

13 Han Y, Zhang PJ, Chen T, Yum SW, Pasha T and Furth EE: Connexin 43 expression increases in the epithelium and stroma along the colonic neoplastic progression pathway: implications for its oncogenic role. Gastroenterol Res Pract 10: 561719, 2011.

14 Husøy T, Knutsen HK, Cruciani V, Olstørn HB, Mikalsen SO, Løberg EM and Alexander J: Connexin 43 is overexpressed in $A p c^{M i n /+-}$ mice adenomas and colocalises with COX-2 inmyofibroblasts. Int J Cancer 116: 351-358, 2005.

15 Aronica E, Gorter JA, Jansen GH, Leenstra S, Yankaya B and Troost D: Expression of connexin 43 and connexin 32 gapjunction proteins in epilepsy-associated brain tumors and in the perilesional epileptic cortex. Acta Neuropathol 101: 449-459, 2001.

16 Rubio CA, Grimelius L, Lindholm J, Hamberg H, Porwit A, Elmberger G, Höög A, Kanter L, Eriksson E, Stemme S, Orrego A, Saft L, Petersson F, De La Torre M, Ekström C, Astrom K, Rundgren A, Djokic M, Chandanos E, Lenander C, Machado M, Nilsson P and Mattsson L: Reliability of the reported size of removed colorectal polyps. Anticancer Res 26: 4895-4899, 2006.
17 Hamilton SR, Rubio CA and Vogelstein V: Carcinoma of the colon and rectum. In: Classification of Tumours of the Digestive System. Bosman FT, Carneiro F, Hruban RH (eds.). Thise ND, Geneve, WHO Press, 2010.

18 Kanczuga-Koda L, Koda M, Sulkowski S, Wincewitz A, Zalewski B and Sulkowska M: Gradual loss of functional gap junction within progression of colorectal cancer - a shift from membranous CX32 and CX43 expression to cytoplasmic pattern during colorectal carcinogenesis. In Vivo 24: 101-108, 2010.

19 Kanczuga-Koda L, Sulkowski S, Lenczewski A, Koda M, Wincewicz A, Baltaziak $M$ and Sulkowska M: Increased expression of connexins 26 and 43 in lymph node metastases of breast cancer. J Clin Pathol 59: 429-433, 2006.

20 Dang X, Doble BW and Kardami E: The carboxy-tail of connexin-43 localizes to the nucleus and inhibits cell growth. Mol Cell Biochem 242: 35-38, 2003.

21 Martins-Marques T, Catarino S, Zuzarte M, Marques C, Matafome $\mathrm{P}$, Pereira $\mathrm{P}$ and Girão $\mathrm{H}$ : Ischaemia-induced autophagy leads to degradation of gap junction protein connexin 43 in cardiomyocytes. Biochem J 467: 231-245, 2015.

22 Lytras T, Nikolopoulos G and Bonovas S: Statins and the risk of colorectal cancer: An updated systematic review and metaanalysis of 40 studies. World J Gastroenterol 20: 1858-1870, 2014.

23 Novo R, Freire CM, Felisbino S, Minicucci MF, Azevedo PS, Zornoff LA and Paiva SA: Smoking is associated with remodeling of gap junction in the rat heart: Smoker's paradox explanation? Arq Bras Cardiol 100: 274-280, 2013.

24 Habermann H, Ray V, Habermann W and Prins GS: Alterations in gap junction protein expression in human benign prostatic hyperplasia and prostate cancer. J Urol 167: 655-660, 2002.

25 Sawey MJ, Goldschmidt MH, Risek B, Gilula NB and Lo CW: Perturbation in connexin 43 and connexin 26 gap-junction expression in mouse skin hyperplasia and neoplasia. Mol Carcinog 17: 49-61, 1996.

26 Andrade-Rozental AF, Rozental R, Hopperstad MG, Wu JK, Vrionis FD and Spray DC: Gap junctions: the "kiss of death" and the "kiss of life". Brain Res Rev 32: 308-315, 2000.

27 Decrock E, Vinken M, De Vuyst E, Krysko DV, D'Herde K, Vanhaecke T, Vandenabeele P, Rogiers V and Leybaert L: Connexin-related signaling in cell death: to live or let die? Cell Death Differ 16: 524-536, 2009.
Received July 3, 2016

Revised August 29, 2016

Accepted September 2, 2016 